\title{
Magnetic gradiometry using frequency-domain filtering
}

Ream, Jodie B.; Weiss, Benjamin P.; Oran, Rona; Raymond, Carol A.; Polanskey, Carol A.; Wenkert, Daniel D.; Elkins-Tanton, Linda T; Hart, Richard A; Russell, Christopher T; Merayo, Jose M G

Published in:

Measurement Science and Technology

Link to article, DOI:

10.1088/1361-6501/ac2e2e

Publication date:

2021

Document Version

Publisher's PDF, also known as Version of record

Link back to DTU Orbit

Citation (APA):

Ream, J. B., Weiss, B. P., Oran, R., Raymond, C. A., Polanskey, C. A., Wenkert, D. D., Elkins-Tanton, L. T., Hart, R. A., Russell, C. T., \& Merayo, J. M. G. (2021). Magnetic gradiometry using frequency-domain filtering. Measurement Science and Technology, 33(1), [015104]. https://doi.org/10.1088/1361-6501/ac2e2e

\section{General rights}

Copyright and moral rights for the publications made accessible in the public portal are retained by the authors and/or other copyright owners and it is a condition of accessing publications that users recognise and abide by the legal requirements associated with these rights.

- Users may download and print one copy of any publication from the public portal for the purpose of private study or research.

- You may not further distribute the material or use it for any profit-making activity or commercial gain

- You may freely distribute the URL identifying the publication in the public portal 
PAPER • OPEN ACCESS

\section{Magnetic gradiometry using frequency-domain} filtering

To cite this article: Jodie B Ream et al 2022 Meas. Sci. Technol. 33015104

View the article online for updates and enhancements.
You may also like

A method of rotating accelerometer gravity gradiometer for centrifugal gradient detection

Mingbiao Yu and Tijing Cai

Ultra-sensitive electrostatic planar acceleration gradiometer for airborne geophysical surveys

Karim Douch, Bruno Christophe, Bernard Foulon et al.

Superconducting sensors and methods in geophysical applications

R Stolz, M Schmelz, V Zakosarenko et al. 


\title{
Magnetic gradiometry using frequency-domain filtering
}

\author{
Jodie B Ream ${ }^{1, *}$, Benjamin P Weiss ${ }^{1}$, Rona Oran ${ }^{1}$, Carol A Raymond ${ }^{2}$, \\ Carol A Polanskey ${ }^{2}$ (D) Daniel D Wenkert ${ }^{2}$, Linda T Elkins-Tanton ${ }^{3}$ (D) Richard A Hart ${ }^{4}$, \\ Christopher T Russell ${ }^{4}$ and Jose M G Merayo ${ }^{5}$
}

${ }^{1}$ Department of Earth, Atmospheric, and Planetary Sciences, Massachusetts Institute of Technology, Cambridge, MA, United States of America

2 Jet Propulsion Laboratory, NASA, Pasadena, CA, United States of America

${ }^{3}$ School of Earth and Space Exploration, Arizona State University, Tempe, AZ, United States of America

${ }^{4}$ Department of Earth Planetary and Space Sciences, University of California Los Angeles, Los Angeles, CA, United States of America

5 Technical University of Denmark, Ørsted·DTU Measurement and Instrumentation Systems, Lyngby, Denmark

E-mail: jbream@mit.edu

Received 15 June 2021, revised 24 September 2021

Accepted for publication 8 October 2021

Published 2 November 2021

\begin{abstract}
Accurate measurements of ambient planetary and interplanetary magnetic fields using spacecraft magnetometers typically require accounting for interfering magnetic fields generated by the flight system (FS). The most common method for removing FS-generated time-variable magnetic fields is narrow-band and low-pass filtering of magnetic field data in the frequency domain. However, if fluctuations in the ambient field contain frequencies overlapping those in the FS field, it can be difficult to construct a filter that will not affect both signals. Here we present an alternate method for removing FS time-variable signatures from magnetic field measurements. For spacecraft that make use of a magnetic gradiometer (i.e. with two or more instruments on a boom at different distances from the center of the spacecraft), the dominant frequencies in the FS field can be identified using spectra of the differenced field components. The amplitudes of the FS field at those frequencies can then be suppressed without removing spectral peaks present in the ambient field. We demonstrate the successful application of this method, referred to as gradiometry peak suppression, both to modeled data sets and to $128 \mathrm{~Hz}$ Venus Express magnetometer data.
\end{abstract}

Supplementary material for this article is available online

Keywords: gradiometry, spectral analysis, magnetic fields

(Some figures may appear in colour only in the online journal)

\footnotetext{
* Author to whom any correspondence should be addressed.
} 


\section{Introduction}

Spacecraft magnetic field measurements are fundamental for the study of the solar system magnetism including planetary dynamos, planetary induction fields, crustal remanence, the interplanetary magnetic field (IMF) and space weather. However, a key challenge in making such observations is that the field measured by spacecraft magnetometers includes both the ambient field plus magnetic fields generated by the fight system. In many cases the flight system (FS) fields can be the dominant noise if not removed.

Major sources of FS field contamination are the alternating current (AC) fields (i.e. having frequency, $f$, typically $>0.01 \mathrm{~Hz}$ ) generated by permanently magnetized and/or highcurrent components such as stepper motors, reaction wheels, communication components, and heaters [1]. This contamination can be reduced by mounting the magnetometer sensors on a boom at the end of which the FS field will be attenuated and also by implementing a magnetic cleanliness program during mission design to limit the magnetic moments of individual FS components. FS AC fields can also be mitigated following data acquisition using a variety of computation techniques. If the spacecraft is spinning, the FS fields and the ambient fields can be distinguished during post-processing on the ground since the ambient field will be modulated at the spin frequency but the FS field will remain stationary. For non-spinning spacecraft, separating the FS and ambient field fluctuations is less straightforward. One common method for removing $\mathrm{AC}$ fields from such datasets is to filter them in the frequency domain based on a priori constraints on the frequency-content of the FS field signatures. Other methods include time-averaging measurements taken by multiple sensors and time-averaging measurements taken by a single sensor to eliminate high-frequency FS fields [2-8].

For spacecraft that carry only one magnetometer sensor, filtering can only be accomplished by using spacecraft ancillary data (e.g. the timing of changes of state of FS components) along with a priori knowledge of the frequencies generated by the different subsystems and components on the spacecraft. Information about the expected FS fields can be provided by ground-based testing prior to launch as well as from in-flight calibration activities. The difficulty in accurately identifying FS fields is that they are often composed of multiple signals with different amplitudes and frequencies originating from multiple components at different distances and directions from the sensor. As a result, it is difficult to isolate contributions from the FS and ambient field at a given magnetometer sensor location. This is particularly challenging when the FS fields have frequencies similar to those present in the ambient field, making it difficult to design a filter that targets only the FS fields.

For spacecraft that carry two or more magnetometer sensors mounted at different distances from the spacecraft bus, gradiometry methods can be used to remove spacecraft generated fields from the measurements. Magnetic gradiometry has been a staple in spacecraft magnetometry investigations since it was first developed by Ness (1971) for Mariner 10 [9]. This method uses simultaneous measurements from two or more sensors on a boom to remove the FS field by measuring the field gradient. In this configuration, each sensor measures a combination of the ambient field, $\vec{B}_{\mathrm{amb}}$, and the FS field at the location of the sensor, $\vec{B}_{\mathrm{FS}}\left(\vec{r}_{k}\right)$, where $\vec{r}_{k}$ is the position of the $k^{\text {th }}$ sensor in the spacecraft coordinate frame. Typically, the distance to the ambient field source is much larger than the separation between the two sensors. As a result, the ambient field can be recognized by the fact that it is the same at the sensors while the FS field is weaker at the more distal sensors. It is important that the gradiometer sensors are time synchronized to a fraction of the time between measurements in order to accurately determine the differenced field (e.g. to a typical accuracy of a few microseconds). If there is a temporal offset in the measurements some corrections can be made in post processing, but the efficacy of the gradiometry methods will be reduced.

Here we consider the specific configuration of two sensors mounted along a single boom, with an outboard sensor $(k=2)$ at the end of the boom and the inboard sensor $(k=1)$ closer to the spacecraft bus. Each sensor measures the three orthogonal components of the magnetic field $(j=1,2,3)$. The coupling coefficients are defined as $\alpha_{i j}=\frac{B_{i, \text { out }}}{B_{j, \text { in }}}$, where $B_{i, \text { out }}$ is the $i$ th component of the observation at the outboard sensor and $B_{j \text {,in }}$ is the $j$ th component of the observation at the inboard sensor. The coupling coefficients form a diagonal matrix whose entries for $i \neq j$ are 0 . In the case where there the field has a single multipole term, all entries along the diagonal are identical. However, for fields with more than one multipole term $\alpha_{11} \neq \alpha_{22} \neq \alpha_{33}$. The latter includes cases with multiple additive FS sources, as well as cases where the field sources do not lie along the boom axis. The differenced field is given by

$$
\begin{aligned}
\Delta \vec{B} & \equiv \vec{B}_{\text {in }}-\vec{B}_{\text {out }} \\
& =\vec{B}_{\mathrm{FS}}\left(r_{\text {in }}\right)+\vec{B}_{\mathrm{amb}}-\left(\vec{B}_{\mathrm{FS}}\left(r_{\text {out }}\right)+\vec{B}_{\mathrm{amb}}\right) \\
& =\vec{B}_{\mathrm{FS}}\left(r_{\text {in }}\right)-\vec{B}_{\mathrm{FS}}\left(r_{\text {out }}\right) .
\end{aligned}
$$

From (1) it can be seen that the differenced field contains only the field generated by the spacecraft because the ambient field terms cancel out.

The spacecraft gradiometry equations developed by Ness [9] give estimates for the FS field at the inboard and outboard sensors, $\vec{B}_{\mathrm{FS} \_ \text {in }}^{\text {sst }}$ and $\vec{B}_{\mathrm{FS} \_ \text {out }}^{\text {est }}$, and the ambient field $\left(\vec{B}_{a m}^{e s t}\right)$ as

$$
\begin{aligned}
\vec{B}_{\mathrm{FS} \_ \text {in }}^{\text {est }} & =(1-\boldsymbol{\alpha})^{-1}\left[\vec{B}_{\text {in }}-\vec{B}_{\text {out }}\right] \\
\vec{B}_{\mathrm{FS} \_ \text {out }}^{\text {est }} & =\boldsymbol{\alpha}(1-\boldsymbol{\alpha})^{-1}\left[\vec{B}_{\text {in }}-\vec{B}_{\text {out }}\right] \\
\vec{B}_{\text {am }}^{\text {est }} & =(1-\boldsymbol{\alpha})^{-1}\left[\vec{B}_{\text {out }}-\boldsymbol{\alpha} \vec{B}_{\text {in }}\right]
\end{aligned}
$$

where $\boldsymbol{\alpha}$ is the coupling matrix discussed above. These equations can be used to correct for direct current (DC) (i.e. time-stationary) fields as well as step changes (i.e. abrupt changes in the FS DC field, resulting from a component being turned on and off) when these fields are strong compared to the intrinsic instrument noise. However, to accurately identify the coupling coefficients, the FS field magnitude must be several times larger than the instrument noise. When the magnitude of 
the FS field is comparable to the instrument noise, uncertainty in the coupling coefficients can cause significant errors in the removal of the FS field components [5, 9].

This becomes especially apparent when using gradiometry to remove a spacecraft-generated AC field. Calculating the spacecraft field on a point-by-point basis leads to large uncertainties around the nodes of the FS AC field oscillations because the FS field contributions are comparable to the instrument noise at those times. However, the frequency content and the timing of the FS contributions with magnitudes comparable to the instrument noise can be identified in the differenced field even if the coupling coefficients, and the true amplitude of the FS field, cannot be accurately determined.

Here we describe a method for cleaning FS fields from magnetometry data by which specific frequencies, referred to as spectral peaks, are identified in the power spectrum of the differenced field and then suppressed in the power spectra of the observed fields at each sensor. This method, which we call frequency-domain gradiometry filtering (or, more briefly, gradiometry peak suppression) enables the identification and suppression of the FS AC field while leaving the ambient field fluctuations largely intact. This method of separating spectral peaks is used in numerous signal processing applications such as removing or enhancing emissions from specific molecules in nuclear magnetic resonance spectroscopy [10-12], removing digital artifacts due to transmission errors from digital images [13], and correcting exposure thresholds for specific regions in medical radiography images [14].

This manuscript is organized as follows. In section 2, we describe how to use frequency-domain gradiometry filtering with a magnetic gradiometer to identify and remove AC signals generated by FS components. In section 3, we describe synthetic datasets used to test the gradiometry peak suppression method. In section 4, we discuss the results of application of gradiometry peak suppression to the synthetic datasets. In section 5, we apply the method to Venus Express (VEX) data and compare the results to published measurements. Finally, in section 6 , we discuss the applications of peak suppression gradiometry to existing and future missions and data and also discuss its limitations.

\section{Method}

The gradiometry peak suppression method identifies one or more discrete frequencies contaminated by FS fields using spectra of $\Delta B_{x}, \Delta B_{y}$, and $\Delta B_{z}$ and then suppresses the power at those frequencies in the spectra of the inboard and outboard sensor measurements. As such, the first step is to calculate the differenced field $(\Delta \vec{B})$. This can be accomplished using either the individual components, $\Delta B_{x}, \Delta B_{y}$, and $\Delta B_{z}$, or the total differenced field given by $|\Delta B|=\left|B_{\text {in }}\right|-\left|B_{\text {out }}\right|$. Our preferred method is to use the total differenced field since the amplitude of the fluctuations in the total field is more likely to exceed the predetermined threshold for detection than each of the three field components individually for FS sources that do not lie along the boom axis.
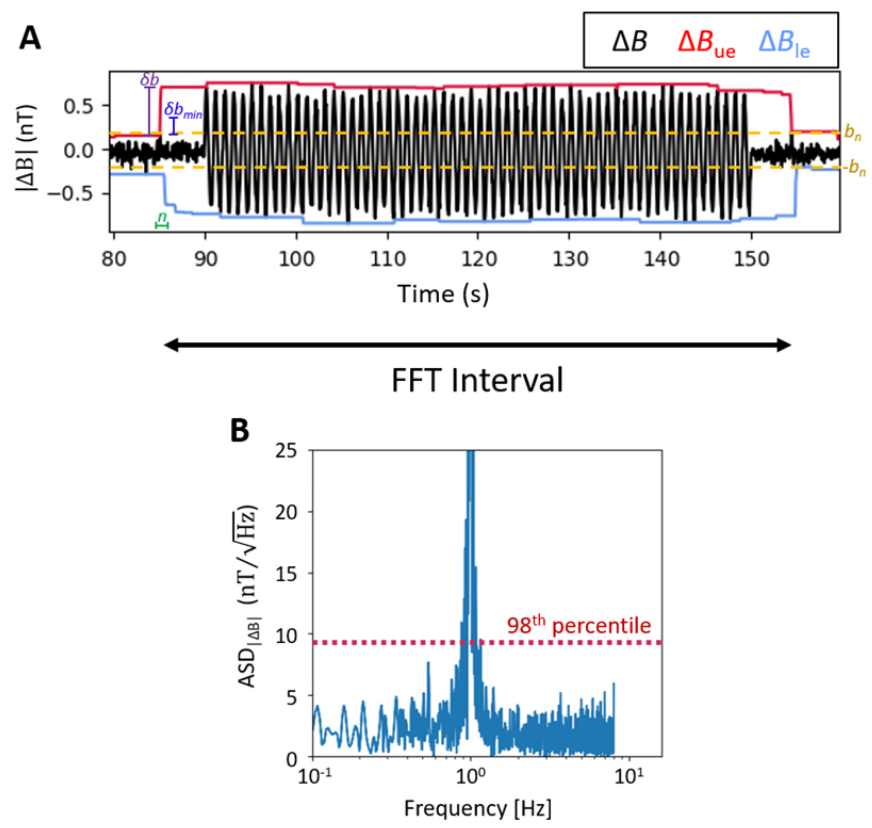

Figure 1. Schematics showing process for identifying flight system frequencies for peak suppression. (A) Time interval selection process. Shown are time series for $\Delta \mathrm{B}$ (black), $\Delta B_{\mathrm{ue}}$ (red) and $\Delta B_{\mathrm{le}}$ (blue) for a flight system AC signal. The value of $n$ (green) is defined for the beginning of the change in the envelope. Yellow dashed lines show the envelope of the instrument noise $\pm b_{n}$. The FFT interval (black arrow) is defined by the times of the changes in $\Delta B_{\text {ue }}$ and $\Delta B_{\text {le }}$. (B) Peak identification process. Shown is the Amplitude Spectral Density (ASD) of the time series in panel A. The 98th percentile line is shown as the red dotted line.

Once the differenced field is calculated, the next step is to determine when a FS AC field is above the noise floor, $b_{n}$, which we take as the $3 \sigma$ instrument noise (see below). To accomplish this, we identify the upper and lower envelopes of the differenced field. The upper envelope $\left(\Delta B_{\mathrm{ue}}\right)$ and lower envelope $\left(\Delta B_{\text {le }}\right)$ are determined by taking a rolling maximum and minimum of the differenced field. Similar to a running average, the maximum and minumum values are determined for a sliding window of a predetermined length, $\delta t$. An example timeseries of $\Delta B, \Delta B_{\text {ue }}$, and $\Delta B_{\text {le }}$ is shown in figure 1(A). Accurately identifying the FS magnetic field signatures requires, at a minimum, one full period for each $\mathrm{AC}$ field contribution, such that the value of $\delta t$ depends on the frequency range of the FS generated AC fields.

FS AC signals are identified as simultaneous changes in $\Delta B_{\text {ue }}$ and $\Delta B_{\mathrm{le}}$. Interval identification is determined based on two parameters, $\delta b$ and $n$. $\delta b$ is defined as the absolute value of the change in $\Delta B_{\mathrm{ue}}$ and $\Delta B_{\mathrm{le}}$ at the beginning of the envelope, and $n$ is a specified number of time steps over which the change in the envelope must occur. Intervals are selected for fluctuation removal when $\delta b$ is larger than a predefined threshold, $\delta b_{\min }$, over a specified number of time steps, $n$, such that $\delta b / n \geqslant \delta b_{\min } / n$.

The field magnitude threshold, $\delta b_{\text {min }}$, indicates the smallest change in the envelopes that can result in a time interval being selected. If a FS AC field has an amplitude comparable to 
the instrument noise, the signal can be identified in the power spectra, but if the amplitude is lower than the instrument noise, the frequency of the fluctuations will be too distorted to accurately identify. As a result, $\delta b_{\min }=b_{n}$ is a reasonable initial threshold.

The other adjustable parameter, $n$, depends on how rapidly the amplitude of AC FS signals change in time (e.g. time required for a component to turn on or off and whether there is a ramp-up period of gradually increasing power) (figure 1(A)). For most components, the change in field will likely be abrupt, occurring over a small fraction of a second, such that a relatively small number of timesteps can be used to identify the edges of the envelope [7].

The next step is to calculate the fast Fourier transform (FFT) of each component of $\vec{B}_{\text {in }}, \vec{B}_{\text {out }}$, and $\Delta \vec{B}$ for the interval identified using envelope changes. Prior to calculating the FFT, we apply a Kaiser $(\beta=10)$ windowing function [15] to the AC interval; this minimizes the energy in the fluctuations at the edges of the time interval and avoids introducing a heavyside function in the time series that would introduce artificial fluctuations in the spectral analysis. In addition, following standard FFT techniques, the dataset is mirrored at both ends (e.g. the whole interval is temporally reversed and appended to both the beginning and the end of the original interval) to minimize edge effects associated with the finite time interval.

Peaks in the FS field are identified in the spectrum of the $\Delta B$ as frequencies with amplitudes greater than the 98th percentile for the interval figure 1(B). This threshold can be modified based on the spectral profile of the FS field. If the FS field has field contributions at only a few dominant frequencies, then the 98th percentile is a reasonable threshold for identifying the spectral peaks. However, if the FS field has significant contributions over a wide range of frequencies, then a lower threshold can be set. In practice, we have found that the threshold should not be set lower than the 95th percentile in order to avoid selecting the minor peaks that are contributed by instrument noise for removal. Additionally, the lowest frequencies $(f<0.1 \mathrm{~Hz})$ are not included in the peak identification step in order to avoid removing broadband noise at the lower frequencies.

After identifying the frequencies of the spectral peaks in the differenced field, the equivalent field contributions at the selected frequencies in $\vec{B}_{\text {in }}$ and $\vec{B}_{\text {out }}$ are suppressed by setting their amplitude to a small fraction, typically between $1 \%$ and $5 \%$, of the observed amplitude. This value can be adjusted based on the amplitude of the interfering signal with respect to the amplitude of the ambient frequencies. The ultimate goal is to reduce the FS generated spectral peaks to noise levels without generating a delta function in the spectrum.

The final step is to perform an inverse FFT to clean the identified FS AC signature from the time series. The distortion caused by the windowing function is corrected by dividing by the same windowing function that was applied to the time series prior to performing the FFT. This returns reconstructed ambient field time series, $\vec{B}_{\mathrm{amb}}^{\text {est }}$ for the inboard and outboard observations.

\section{Magnetic field models}

We validated the gradiometry peak suppression method by applying it to simulated FS magnetic field datasets. In particular, we compared reconstructed fields derived from these datasets to the simulated ambient fields, $\vec{B}_{\text {amb }}^{\text {actual }}$, to determine the error in the gradiometry peak suppression calculations given by $\varepsilon=\vec{B}_{\text {amb }}^{\text {actual }}-\vec{B}_{\text {amb }}^{\text {est }}$.

We considered two field models. Model 1 (supplementary material Model1.csv available online at stacks.iop.org/MST/ $33 / 015104 /$ mmedia) was designed to test the application of our method on a field model containing multiple AC signatures from different FS components that overlap in time. Model 2 (supplementary material Model2.csv available online at stacks.iop.org/MST/33/01510/mmedia) was used to determine the lower limit on the frequency difference between FS and ambient field frequencies before the gradiometry peak suppresison method can no longer reliably identify and remove the FS-generated fields. Both simulated datasets were developed for a virtual spacecraft with two sensors placed on a boom at $1.4 \mathrm{~m}$ and $2.1 \mathrm{~m}$ above the top deck of the spacecraft bus. Simulated observations at each of the two sensors include an ambient field, $\vec{B}_{\text {amb }}$, FS-generated AC fields, $\vec{B}_{\text {FS_in }}$ and $\vec{B}_{\text {FS_out }}$, and instrument noise, $\vec{B}_{\text {noise_in }}$ and $\vec{B}_{\text {noise_out }}$. The total field at each sensor is therefore:

$$
\begin{aligned}
\vec{B}_{\text {in }}(t) & =\vec{B}_{\mathrm{FS} \_ \text {in }}(t)+\vec{B}_{\mathrm{amb}}(t)+\vec{B}_{\text {noise_in }}(t) \\
\vec{B}_{\text {out }}(t) & =\vec{B}_{\mathrm{FS} \_ \text {out }}(t)+\vec{B}_{\mathrm{amb}}(t)+\vec{B}_{\text {noise_out }}(t) .
\end{aligned}
$$

The FS and ambient field amplitudes were modeled based on the typical minimum detectable signals for space-based magnetometers $(\sim 1 \mathrm{nT})$. Sensor noise was included as random Gaussian noise with a $3 \sigma$ value of $0.22 \mathrm{nT}$ in each sensor, $\vec{B}_{\text {noise_in }}$ and $\vec{B}_{\text {noise_out }}$.

The models are described in detail in the following sections. The sensor locations, sensor performance and the FS AC fields were chosen to represent those expected for the the upcoming NASA Psyche mission [16, 17] which incorporates dual threeaxis fluxgate magnetometers for measuring the magnetic field of asteroid (16) Psyche.

\subsection{Model 1}

We used Model 1 to test the gradiometry peak suppression method on a realistic FS field containing temporally coincident AC signatures that turn on and off and have varying amplitudes and durations. This $30 \mathrm{~min}$ time series included 20 different synthetic AC sources, modeled as pure sine waves, superposed on actual measurements of the IMF from the Juno magnetometer [18, 19] (figure 2(A)). Each of the AC sources was assigned a randomly-chosen frequency between 0.1 and $16 \mathrm{~Hz}$ and a random location on the spacecraft bus. The start time and duration for each source were also randomly assigned across the 30 min interval. To make the modeled FS field as realistic as possible, no restriction was placed on the onset time for each AC signal. The result is that several AC signals can overlap in a single interval. 


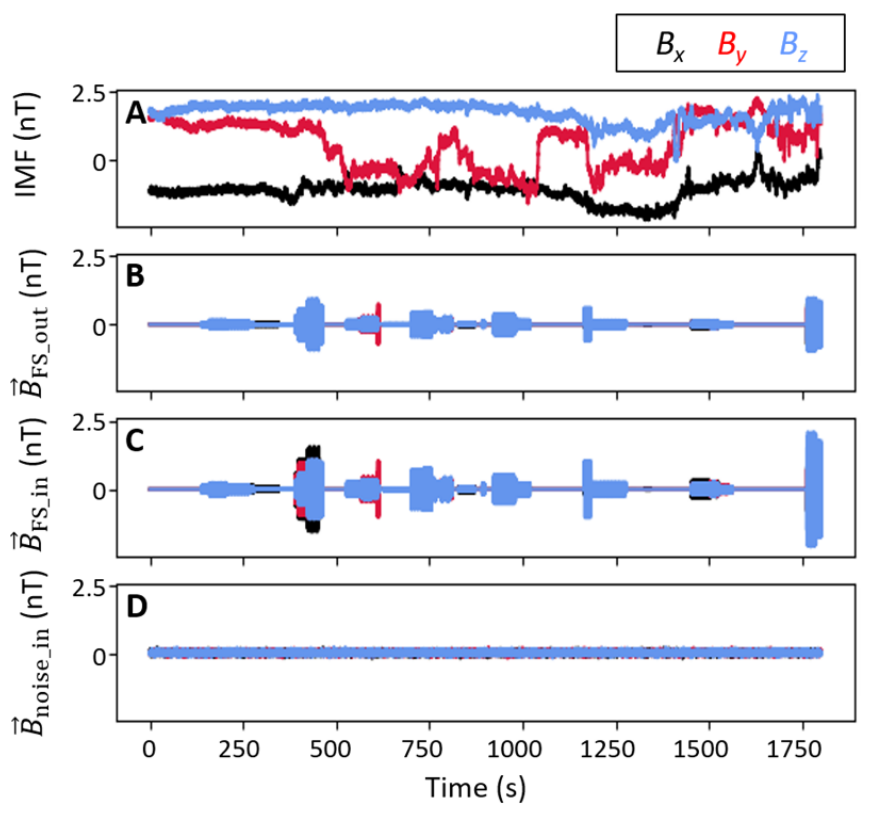

Figure 2. Contributions to the Model 1 field. (A) IMF measurements from the Juno spacecraft. (B) Modelled flight system (FS) AC fields at the outboard sensor. (C) Modelled FS field at the inboard sensor. (D) Instrument noise for the inboard sensor. The colors in each panel indicate $B_{x}$ (black), $B_{y}$ (red) and $B_{z}$ (blue). The abscissa is given as time in seconds measured from the beginning of the dataset. The ordinate gives the magnetic field in $\mathrm{nT}$.

The resulting AC field profiles for the outboard sensor, $\vec{B}_{\mathrm{FS} \text { out }}\left(\right.$ figure 2(B)), and inboard sensor, $\vec{B}_{\mathrm{FS}}$ in (figure 2(C)), and instrument noise, $\vec{B}_{\text {noise_in }}$ and $\vec{B}_{\text {noise_out }}$ (figure 2(D)), are added to the IMF measurements, $\vec{B}_{\mathrm{amb}}=\vec{B}_{\mathrm{IMF}}$ (figure 2(A)). The total field at the inboard and outboard sensors and the differenced are shown in figure 3 . The peak-to-peak amplitude of the AC field at the inboard sensor is $<3 \mathrm{nT}$.

One example of overlapping AC signals is the interval shown in the the shaded box in figure 3 (394.75-453.19 s). The time series and spectra for this interval, which includes several different FS AC sources with frequencies of $0.65 \mathrm{~Hz}$, $1.7 \mathrm{~Hz}, 6.5 \mathrm{~Hz}$ and $7.6 \mathrm{~Hz}$, contribute to the observed field. Details of the total field for each sensor and the differenced field for that interval are shown in figures 4(A)-(C) along with the corresponding amplitude spectra (figures $4(\mathrm{D})-(\mathrm{F})$ ).

\subsection{Model 2}

We used Model 2 to determine the minimum frequency separation $(\delta f)$ required by the gradiometry peak suppression algorithm and the lower limit on the frequencies that can be removed using this method. These relate to frequency resolution in the FFT, which is determined by the sampling frequency and the duration of the intervals selected for FS field removal. With these goals, Model 2 was constructed to contain an ambient field consisting of a single frequency and a single FS source that steps through a range of frequencies around the ambient field frequency. We incorporated FS field sources with frequencies near the lower end of the frequency range where peak suppression is likely to be a viable method for
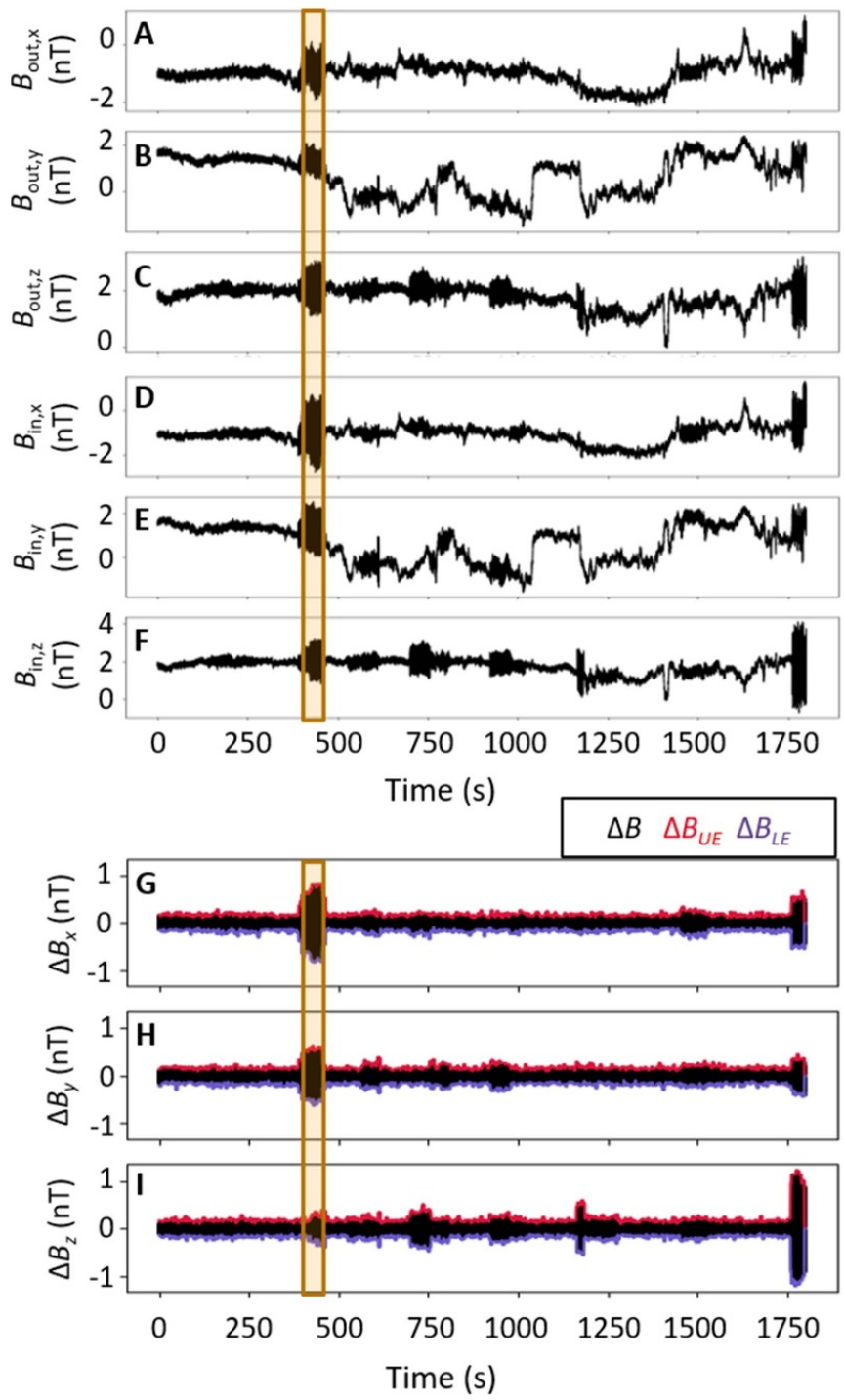

Figure 3. Model 1 observed and differenced fields. Magnetic field components measured by the outboard (A)-(C) and inboard (D)-(F) sensors. (G)-(I) Differenced field along with the upper (magenta) and lower (purple) envelopes constructed using a 2 min rolling maximum and minimum respectively. The highlighted interval indicates the observations used to show the details of the gradiometry peak suppression method.

removing $\mathrm{FS}$ fields, $f \geqslant 0.1 \mathrm{~Hz}$. FS AC frequencies range from 1 to $2.9 \mathrm{~Hz}$ with a step size of $d f=0.1 \mathrm{~Hz}$ and a duration of $60 \mathrm{~s}$ with a gap of $30 \mathrm{~s}$ between each signal. All FS signals have an amplitude of $1.3 \mathrm{nT}$ at the location of the inboard sensor and $0.65 \mathrm{nT}$ at the location of the outboard sensor. The modeled ambient field has a constant frequency $f=1.85 \mathrm{~Hz}$, and an amplitude of $1.5 \mathrm{nT}$. Instrument noise was included in the model as Gaussian noise with a $3 \sigma$ value of $0.22 \mathrm{nT}$.

The resulting observations for the $x$-component of the field are shown in figure 5(A) for the inboard and outboard sensors. In this time interval, the ambient field frequency of $1.85 \mathrm{~Hz}$ lies between two of the FS field frequencies. Figure 5(B) shows the details for the ambient and FS fields for the first $100 \mathrm{~s}$ of the model as indicated by the box in figure 5(A). The FS field during this interval has a frequency of $1 \mathrm{~Hz}$. The coupling 

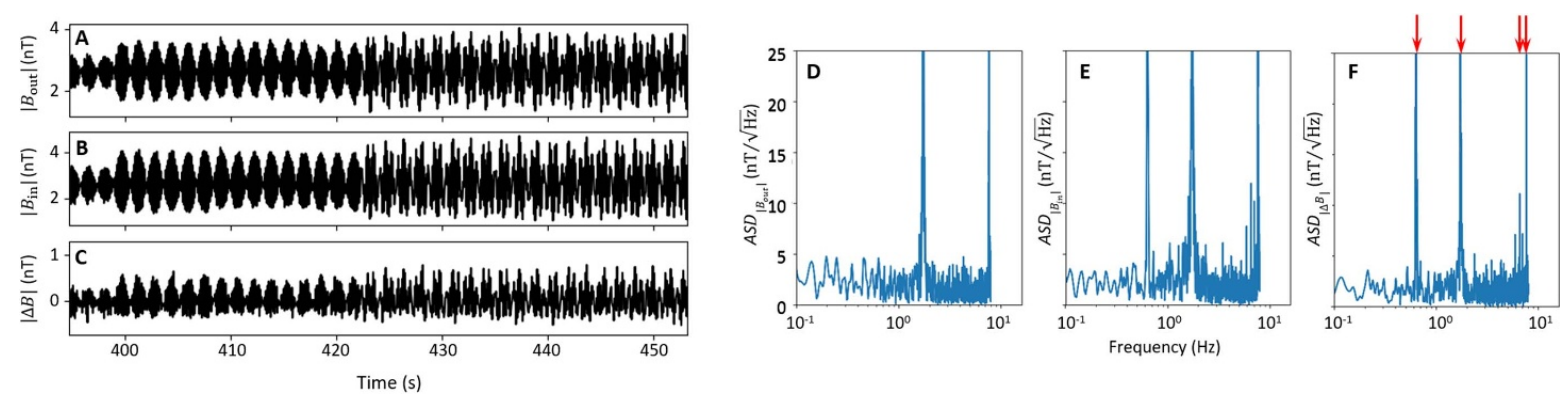

Figure 4. Total fields and their spectral content for the highlighted interval in figure 3. (A) The total outboard field, $\left|B_{\text {out }}\right|$, (B) total inboard field, $\left|B_{\text {in }}\right|$, and (C) total differenced field, $|\Delta B|$, are shown on the left. The ASD is shown on the right for (D) $\left|B_{\text {out }}\right|$, (E) $\left|B_{\text {in }}\right|$, and (F) $|\Delta B|$. The red arrows in panel $\mathrm{F}$ indicate which spectral peaks are above the 98th percentile for the interval.

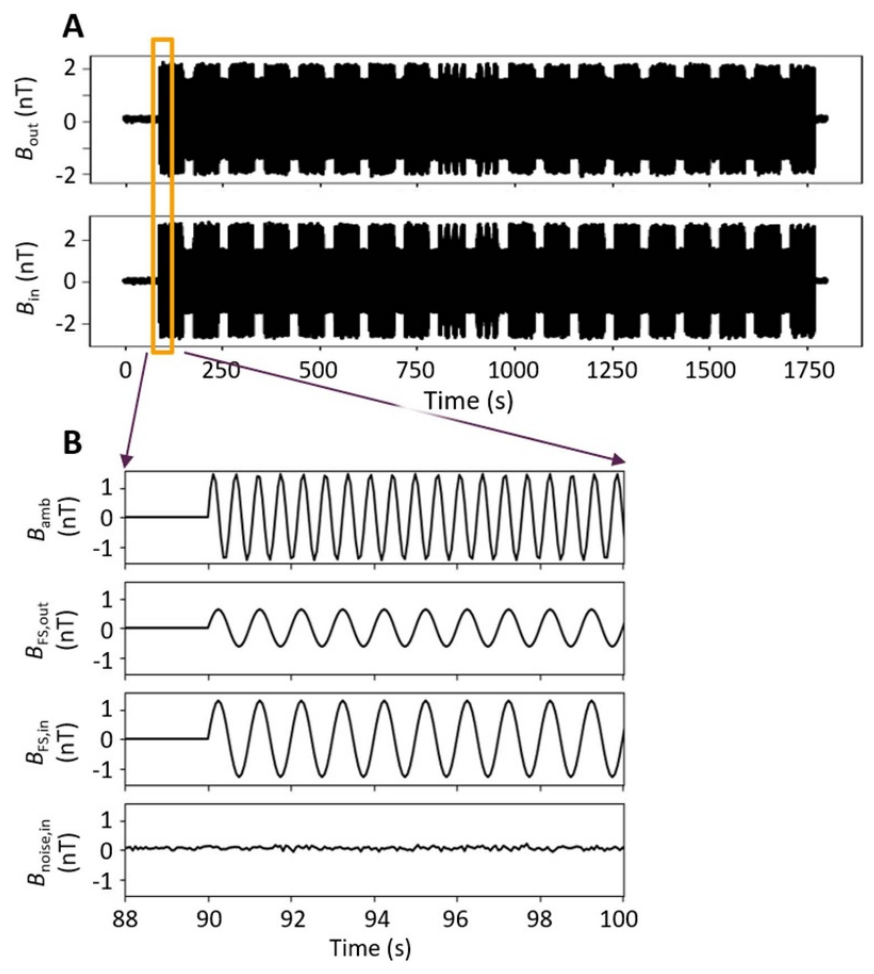

Figure 5. Contributions to Model 2 field. (A) One component $\left(B_{x}\right)$ of the measured field at the outboard and inboard sensors in nT. (B) Initial $100 \mathrm{~s}$ of Model 2 showing the ambient field, flight system field at the outboard and inboard sensors, and the instrument noise.

coefficient for this model is taken to be $\alpha=0.5$. The differenced field for Model 2 is shown in figure 6.

\section{Results}

\subsection{Model 1 results}

To show the details of the peak suppression method described in section 1, we focused on the aforementiond 394.75-453.19 s interval in Model 1 consisting of four FS AC contributions. As noted in section 3.1, the interval is defined by the changes in $\Delta B_{\text {ue }}$ and $\Delta B_{\mathrm{le}}$. The start and stop times for the interval coincide with an AC source switching on or off.

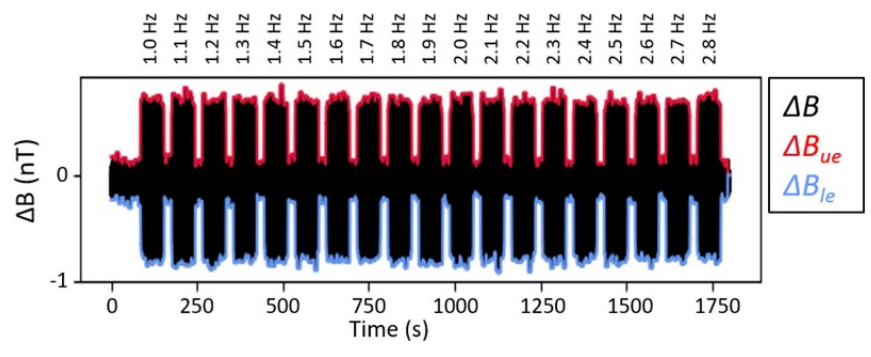

Figure 6. Differenced field for Model 2. The format is the same as the last 3 panels in figure 2. The frequency for each FS field interval is noted at the top of the plot. The upper and lower envelopes are shown in red and blue respectively.

Details of the peak suppression method for this interval are shown in figure 7 . The spectra of the observed signals $\left(\vec{B}_{\text {in }}\right.$ and $\vec{B}_{\text {out }}$ ) contain peaks from both the ambient field and the FS field, while the spectrum of $\Delta \vec{B}$ contains only the peaks generated by the FS (figures 4(D)-(F)). Because there are only four discrete frequencies in the modeled FS field, the dominant peaks in the differenced spectrum were identified as those that have amplitudes above the 98th percentile in the differenced field. In this example, we have used $|\Delta B|$ to determine a single set of peaks for all three components. To suppress the fluctuations in the time series we reduce the amplitude at the selected frequencies in the spectrum to the sensor noise levels by setting it to $1 \%$ of the observed value.

After the differenced peaks were suppressed in the observed signal, an inverse FFT was performed on the corrected spectrum and the distortion caused by the windowing function is corrected. The final result is a time series that does not contain significant fluctuations at the suppressed frequencies. A comparison of the original (i.e. raw) data and cleaned (i.e. estimated) data for Model 1 is shown in figure 8 . The top three panels show the original ambient field, $\vec{B}_{\text {amb }}^{\text {actual }}$, and the reconstructed ambient field, $\vec{B}_{\text {amb }}^{\text {est }}$. The lower three panels show their diffrence, $\varepsilon$. The four FS AC contributions noted in section 3 are identified in $|\Delta B|$ (red arrows in figure $4(\mathrm{~F})$ ). The spectra for the outboard sensor components (figures 7(D)-(F)) show that only the fluctuations at $1.7 \mathrm{~Hz}$ and $7.6 \mathrm{~Hz}$ contribute significantly to the outboard 


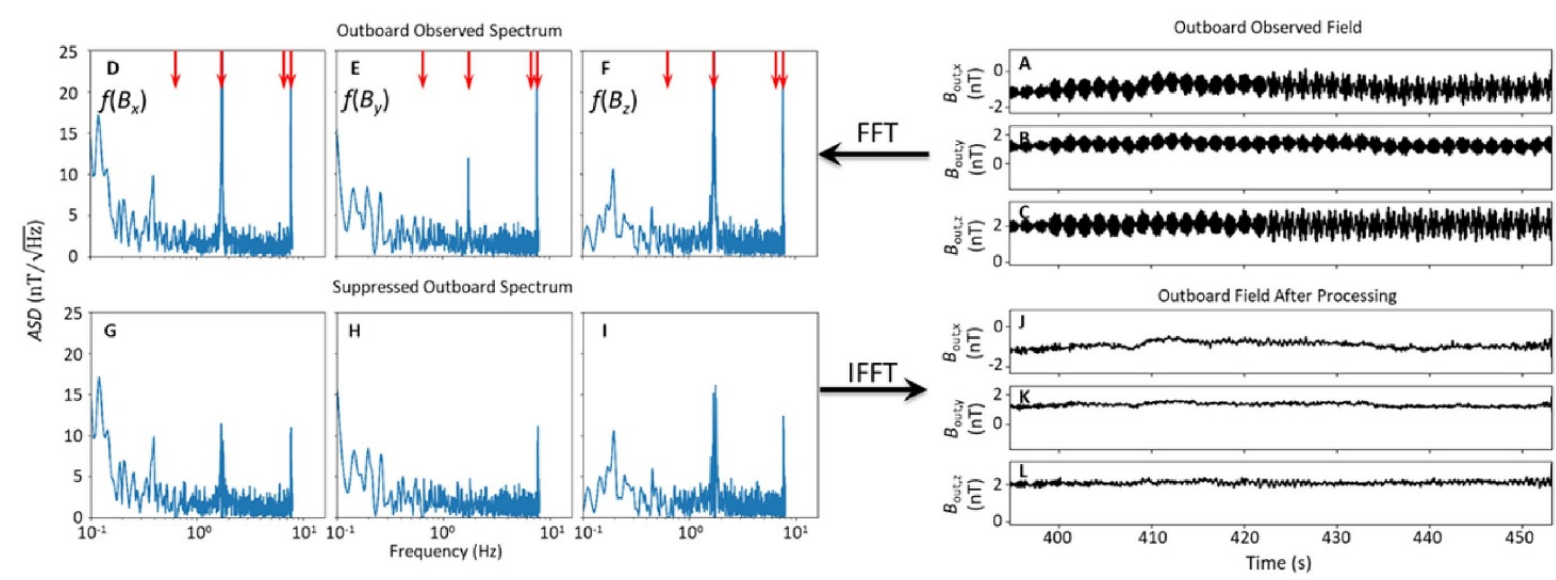

Figure 7. Gradiometry peak suppression method applied to Model 1 data. (A)-(C) Observations for the outboard sensor for $B_{x}, B_{y}$, and $B_{z}$ respectively. (D)-(F) Results from a Fourier transform on the observed data $\left(B_{x}, B_{y}, B_{z}\right)$ at the outboard sensor. (G)-(I) Spectra after the flight system peaks are suppressed. (J)-(L) Cleaned time series for $B_{x}, B_{y}$, and $B_{z}$ respectively plotted on the same scale as the observed field in panels $(\mathrm{A})-(\mathrm{C})$. The red arrows above the observed spectra indicate the frequencies with spectral peaks as observed in the spectrum of $|\Delta B|$ (figure 4(F)) and are suppressed in panels (G)-(I). An inverse Fourier transform (IFFT) using the suppressed spectra results in panels $(\mathrm{J})-(\mathrm{L})$ in the lower right.

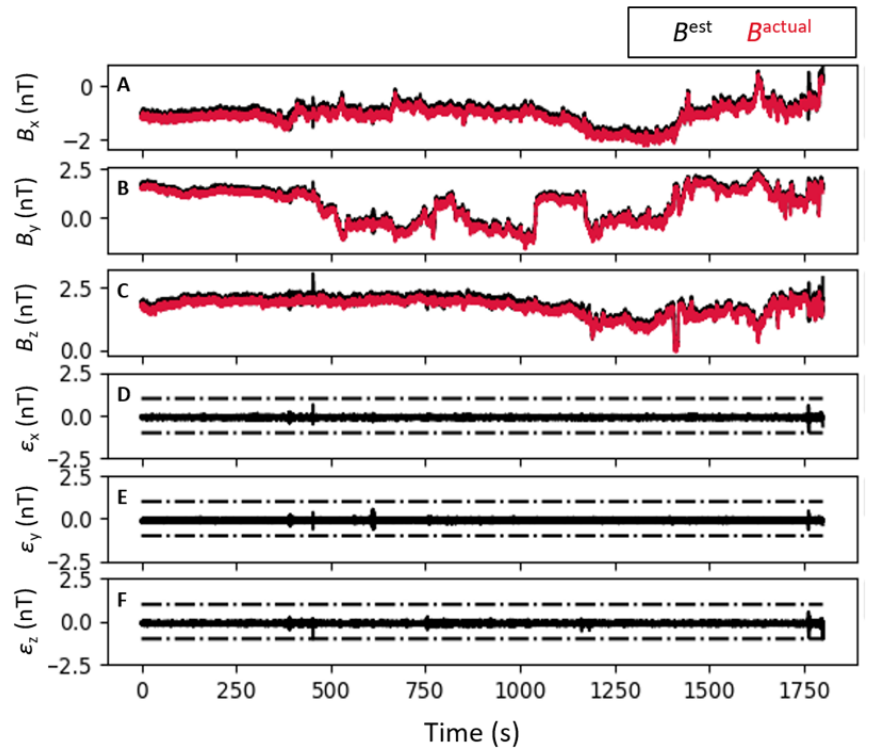

Figure 8. Comparison of raw (actual) and cleaned (estimated) data from Model 1. (A)-(C) The estimated (black) and actual (red) ambient magnetic field $\left(B_{x}, B_{y}, B_{z}\right)$. (D)-(F) Error in the reconstructed field after peak suppression has been applied $\left(\varepsilon_{x}, \varepsilon_{y}, \varepsilon_{z}\right)$. The dot-dash lines are placed at $\pm 1 \mathrm{nT}$.

sensor. The time series (figures 7(A)-(C)) show fluctuations from multiple frequencies for both the $x$ and $z$ components but the y component only shows a minor contribution from the higher frequency fluctuations. Fluctuations from the higher frequency source would have been left in the reconstructed signal if the FS contributions were identified using the components of the differenced field rather than the total differenced field. This is because the power at those frequencies is below the threshold for selection in $\Delta B_{y}$ but not in the other two components. Figures $7(\mathrm{~J})-(\mathrm{L})$ show that the fluctuations are successfully removed from all field components for this

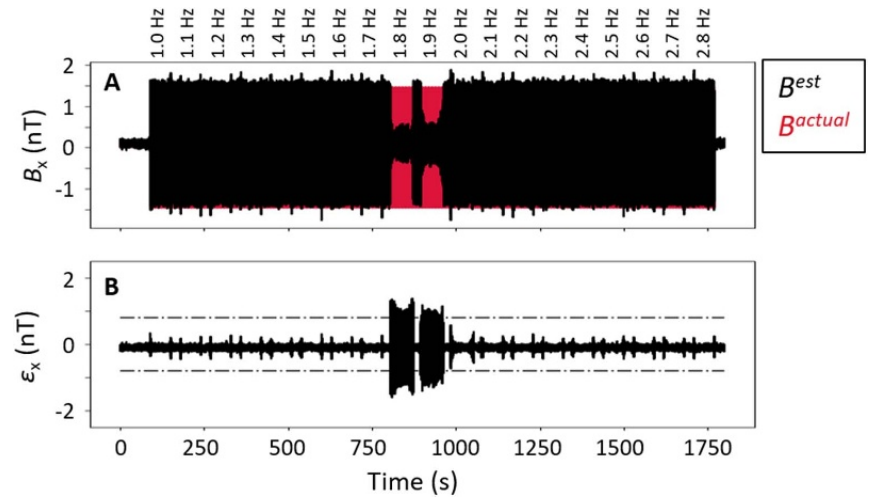

Figure 9. Results from Model 2. Same format as figure 8 for one component of the magnetic field $\left(B_{x}\right)$. The frequency of the FS field is included at the top of the figure for reference. The ambient field frequency is $1.85 \mathrm{~Hz}$.

interval down to the instrument noise levels as described in section 3 .

\subsection{Model 2 results}

Results from Model 2 are shown in figure 9. The only packets that were not cleaned effectively are the two intervals with FS field frequencies separated by $\leqslant 0.05 \mathrm{~Hz}$ from the ambient field frequency of $1.85 \mathrm{~Hz}(1.8 \mathrm{~Hz}$ and $1.9 \mathrm{~Hz})$.

In general, the frequency resolution of the FFT determines the minimum frequency separation between ambient and FS generated fields required to acurately remove only the FS fields. The resolution of the FFT is given by $\delta f=f_{\mathrm{s}} / N_{\mathrm{s}}$ where $f_{\mathrm{s}}$ is the sampling frequency and $N_{\mathrm{s}}$ is the number of samples in the window. Since $N_{\mathrm{s}}=d t \times f_{\mathrm{s}}$, where $d t$ is the duration of the window in seconds, $\delta f=1 / d t$. For example, to obtain a resolution better than $0.05 \mathrm{~Hz}$, the windows should be a minimum of $20 \mathrm{~s}$. This also sets the lower limit on the frequencies that can be removed using peak suppression for a given window, 

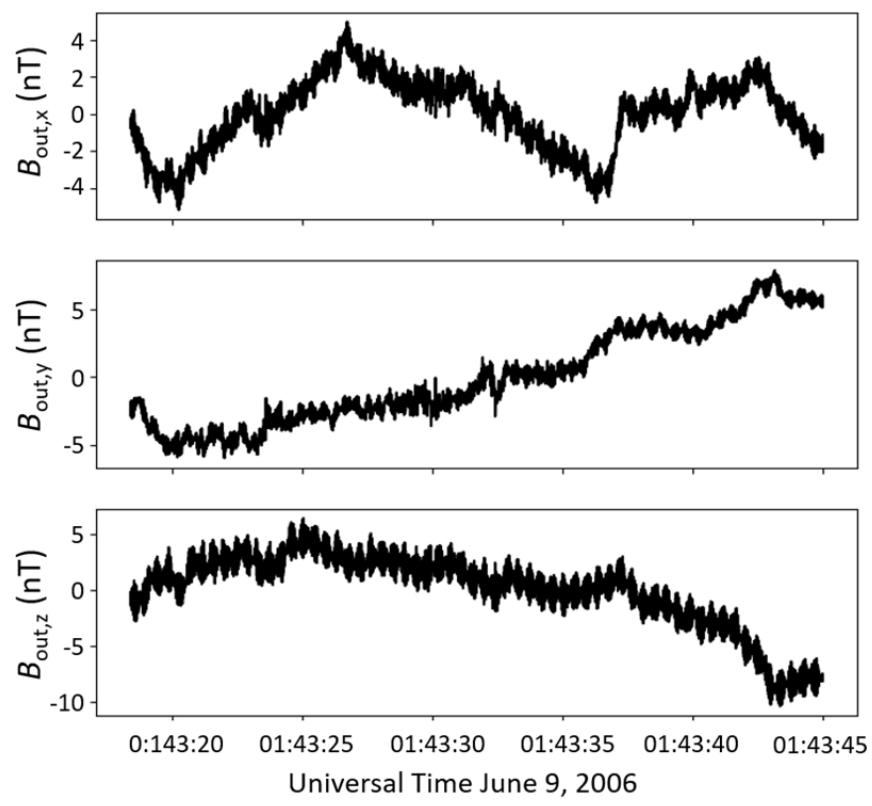

Figure 10. Venus Express outboard sensor measurements $\left(B_{x}, B_{y}, B_{z}\right)$ from 9 June 2006, 0143:18-0143:45 UT before removing the spacecraft field contributions.

which is given by $f_{\min }=2 \times \delta f$. The frequency resolution can be increased by mirroring the beginning and end of the data set by effectively increasing the length of the data set. However, it is possible to artificially enhance the power at some frequencies if only a portion of the data set is included in the mirror. The upper limit on the frequencies that can be identified is set by the measurement Nyquist frequency.

\section{Application to Venus Express data}

\subsection{Venus Express mission and data}

We next apply our method to actual data from the VEX spacecraft mission, which arrived in orbit around Venus in 2006. The goals of the mission included investigation of the plasma environment including lightning on Venus and atmospheric loss mechanisms related to interactions between the Venusian atmosphere and the solar wind [20]. A magnetic gradiometer was included on the spacecraft to help accomplish these goals. The gradiometer consisted of two fluxgate magnetometers on a $1 \mathrm{~m}$ boom. One sensor was mounted at the end of the boom and the other was mounted at the base of the boom, $10 \mathrm{~cm}$ from the spacecraft bus [21]. The instrument had three measurement modes with different frequencies: 1, 32 and $128 \mathrm{~Hz}$. Given that the sensors were positioned so close to the spacecraft, the FS fields observed by the magnetometer sensors were $\sim 200 \mathrm{nT}$ at the outboard sensor [22]. Several methods to remove the FS fields from the observations were developed and the mission was able to report the Venusian fields to within an uncertainty of $\sim 1 \mathrm{nT}[7]$.

During the closest approach to Venus in each orbit, the magnetometer used the $128 \mathrm{~Hz}$ burst mode to study electromagnetic waves thought to be generated by lightning on Venus
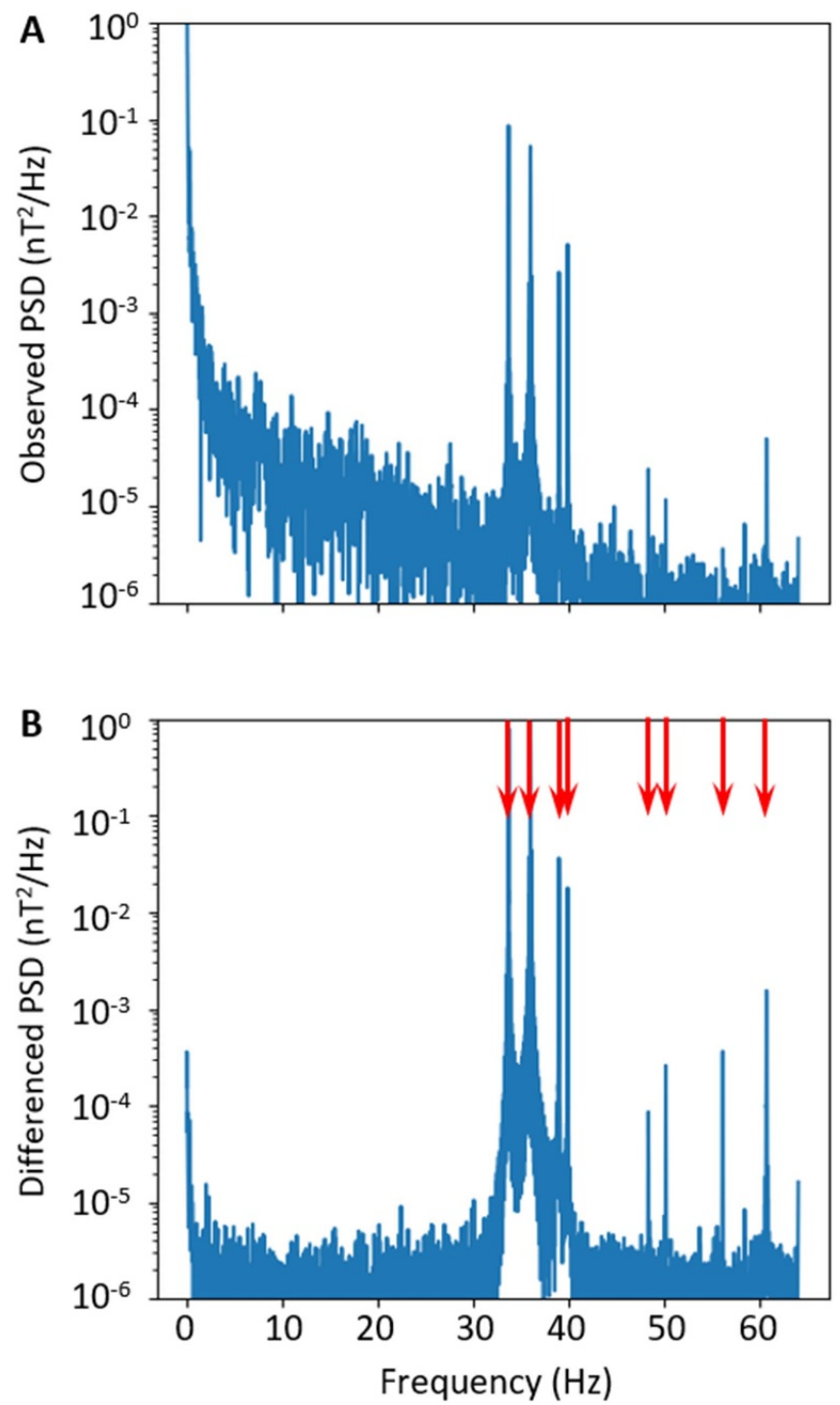

Figure 11. The sum of the powers in the real part of the spectrum for Venus Express observations shown in figure 10. (A) The spectrum for the observed field at the outboard sensor. (B) The spectrum for the differenced field. The red arrows indicate the frequencies with spectral peaks above the 98 th percentile.

$[8,23]$. To test the gradiometry peak suppression method, we selected several VEX orbits previously analyzed by [24]. Outboard sensor measurements from one of those days, 9 June 2006 143:18-143:45 UT, are shown in figure 10. The average value has been removed from each component so that only the fluctuations are shown.

The spectrum for the total field at the outboard sensor and the differenced field are shown in figure 11. Based on the differenced field spectrum, it is apparent that the prominent fluctuations at $32-40 \mathrm{~Hz}$ as well as peaks $48,50,56$, and $60-61 \mathrm{~Hz}$ are FS-generated. These peaks in the FS field are identified in the differenced field spectrum (figure 11(B)) by the red arrows. The difference field, shown in figure 12(A), also shows a lot of interference from the reaction wheels throughout the time series. FS-generated fields have amplitudes on the order of $5 \mathrm{nT}$ at the outboard sensor. 

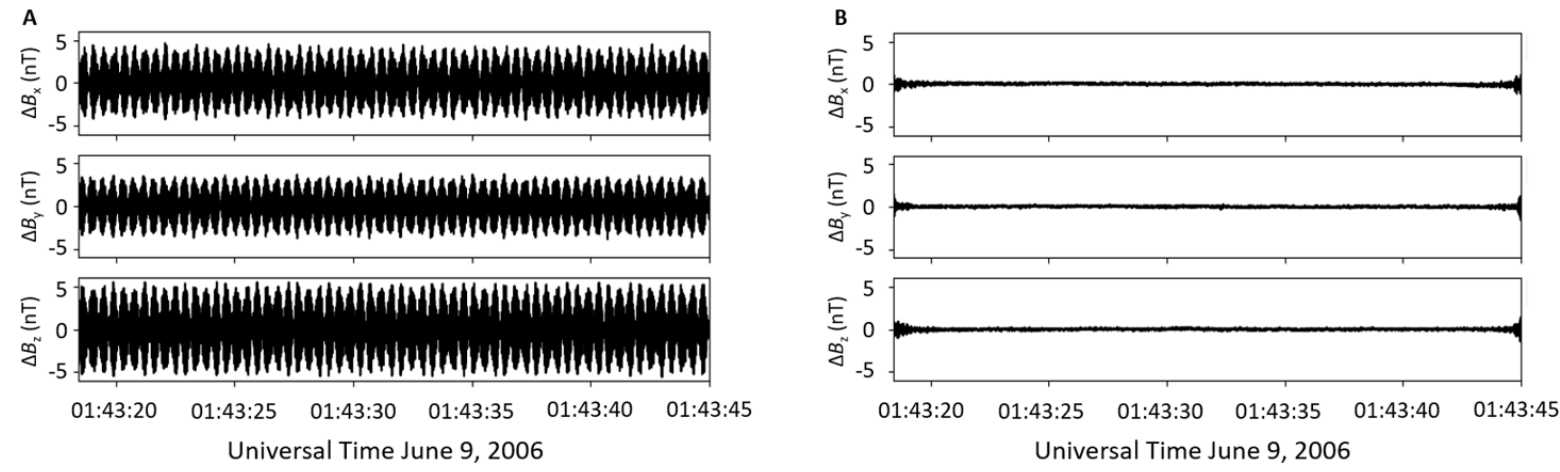

Figure 12. (A) Differenced field ( $\Delta B_{x}, \Delta B_{y}, \Delta B_{z}$ ) for the interval shown in figure 10 before the flight system field has been removed. (B) Differenced field for the same interval as (A) after the flight system field has been removed using gradiometry peak suppression.

\subsection{Method comparison}

The cleaning method used by [24], referred to here as the VEX method, and the gradiometry peak suppression method presented here are very similar in the way they identify the frequencies of the FS fields. Both identify frequencies in the the differenced field spectrum based on whether their power is above a specified threshold. There are two subtle differences in this process. First, the VEX method identifies FS frequencies using the power spectral density (PSD) while the peak suppression method uses the amplitude spectral density (ASD). The effect on the processing is negligible because the differenced field does not contain a significant amount of power in the lower frequencies compared to the FS fluctuations. Second, in the VEX method the spectral content of $\vec{B}_{\text {in }}, \vec{B}_{\text {out }}$ and $\Delta B$, were compared prior to selecting a frequency for removal. Only the frequency bands with power above a predefined threshold in the differenced field and a coupling coefficient above a predefined threshold are selected for removal. In gradiometry peak suppression, the coupling coefficients are not evaluated. All of the frequencies above the 98th percentile in the differenced field are selected and suppressed in the observed spectra for each sensor.

More importantly, the two methods also differ in how the identified FS fields are removed from the observations. In the VEX method, an inverse FFT based on the frequencies selected for removal was used to create a time series of the FS-generated signal. Coupling coefficients were then used to determine the amplitude of the FS signals at the outboard sensor. The time series for the FS-generated field was shifted to match the phase of the fluctuations in the observed time series and then subtracted from the observed field [24].

In comparison, the gradiometry peak suppression method corrects the field in the frequency domain rather than generating a time series for the FS field and using it to make the correction in the time domain. This removes the complication of matching up the phases of the fluctuations in the modeled spacecraft field to the observed field. The VEX method also makes use of the gradiometry equations [9] to determine the amplitude of the FS generated field. This means that the minimum amplitude threshold for identifying a FS field for the VEX method is limited by the need to accurately identify the coupling coefficients. Gradiometry peak suppression only uses the differenced field to identify the frequency of the FS fields rather than calculating the true amplitude of those field contributions. As a result, there is no need to accurately determine the coupling coefficient so the amplitude threshold for FS signals to be identified is reduced compared to more traditional gradiometry methods.

\subsection{Results}

The PSD of the cleaned measurements using two different processing methods are shown in figure 13. The results for the methods show some differences between 30 and $40 \mathrm{~Hz}$. The original VEX results show residual peaks at 33 and $35 \mathrm{~Hz}$, as well as introducing some noise at $31 \mathrm{~Hz}$ that was not present in the measured spectrum. The gradiometry peak suppression method completely removes peaks between $30 \mathrm{~Hz}$ and $39 \mathrm{~Hz}$, but leaves some residual power at $40 \mathrm{~Hz}$ since the power in that frequency is below the 98th percentile for magnitude of the peaks in the differenced field spectrum. The reconstructed time series are very similar for both methods when considering both the wave forms and the noise floor for the results. The red lines show the difference between the results from the VEX method and those from the peak suppression method. During the first $8 \mathrm{~s}(01: 43: 18-01: 43: 26$ UT) the difference in the two results is as large as $0.3 \mathrm{nT}$. For the rest of the time series the differences are $<0.15 \mathrm{nT}$. The differenced field after the FS field has been removed using peak suppression is shown in figures 13(B) and (D). The amplitude fluctuations in the differenced field are reduced from $\sim 5.0 \mathrm{nT}$ to $\sim 0.3 \mathrm{nT}$ after gradiometry peak suppression has been applied (figure 13(D)). There is an improvement of $\sim 10 \mathrm{pT}(14 \%)$ in the RMS noise for the $28-42 \mathrm{~Hz}$ frequency band when using gradiometry peak suppression compared to the results in [24] (figure 13(B)).

Results for the whistler waves analysis for 9 June 2006, 01:43:18-01:43:45 are shown in figure 14. Whistler waves are identified using the ellipticity, whether a signal is right or left hand polarized, and the propagation angle of the fluctuations with respect to the background field $[25,26]$. The coherence is also used to refine the selection of the whistler waves in the data, where the plane with the best coherence between the wave forms in its respective axes is used to identify the 

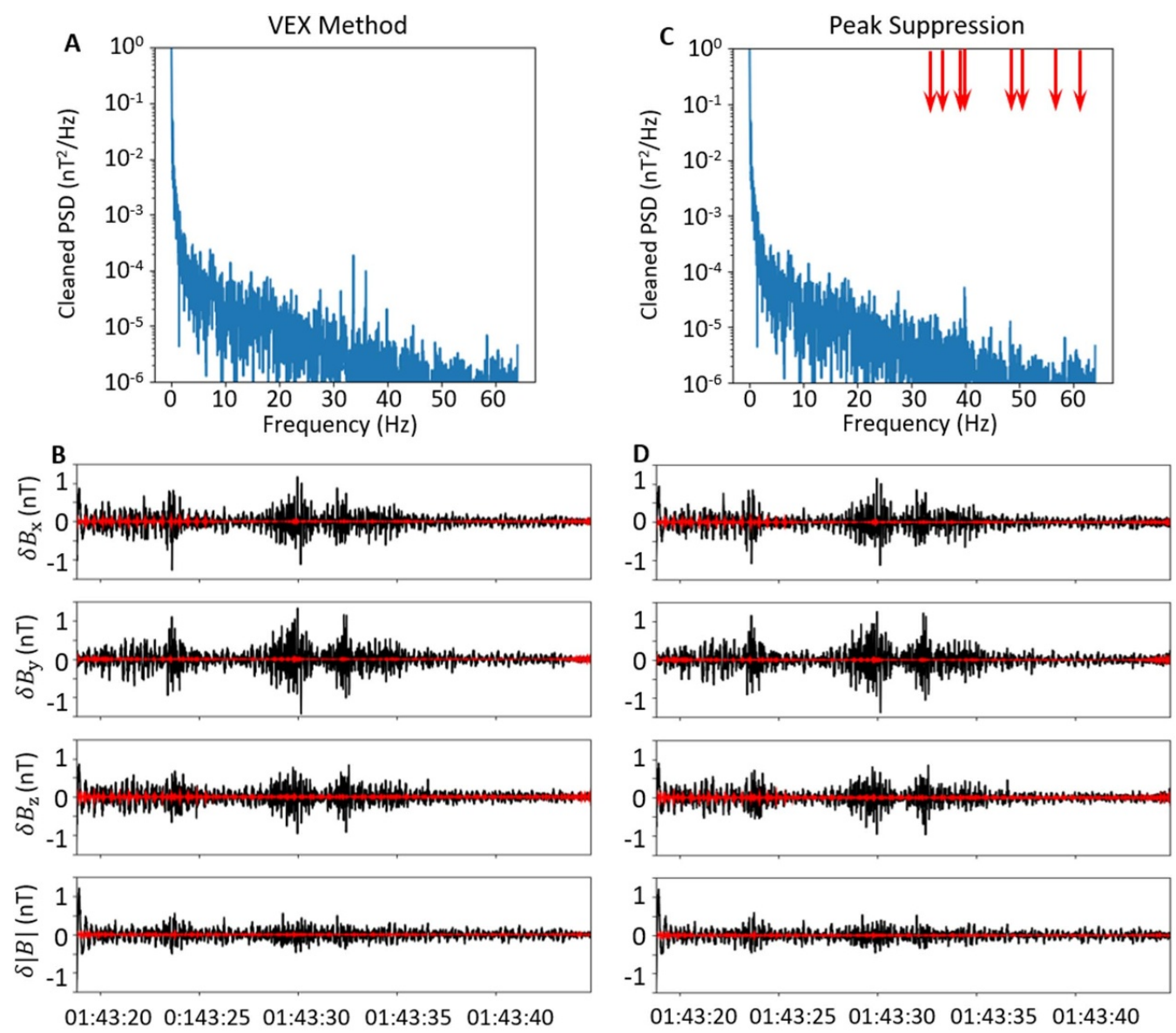

Universal Time June 9, 2006

Figure 13. Application of magnetic gradiometry to Venus Express data from 9 June 2006, 01:43:18-01:43:45. (A), (B) Results presented in [18]. (C), (D) Results from gradiometry peak suppression (this study). (A) and (C) Show the sum of the power in the real part of the spectrum after FS fields have been removed for each method. The red arrows in $(C)$ indicate the peaks selected for removal using the differenced field (figure 11(B)). The corrected field time series for $B_{x}, B_{y}, B_{z}$, and $|B|$ are shown in (B) and (D). Data have been high pass filtered with a $3 \mathrm{~Hz}$ corner frequency. The red line in each panel shows the difference between the results for the two methods for each component.

times and frequencies where the coherence is above a predefined threshold. The ellipticity is calculated from the ratio of the minimum and maximum eigenvalues of the cospectrum, given by $\left(\operatorname{Re}\left[f \cdot f^{*}\right]\right)$, and the propagation angle is determined by calculating the direction of minimum variance for the wave field, $\vec{k}$, and comparing it to the direction of the background magnetic field, $\vec{B}$. The whistler-mode waves are signals with ellipticity $>0.5$, propagation angle $<45^{\circ}$, and coherence $>0.3$ that span at least $1 \mathrm{~s}$ in time and $6 \mathrm{~Hz}$ in frequency. Times are identified when the parameters are above the predefined thresholds as shown by the thick black lines at the botom of each panel and the results are manually checked for false positives [27].

The whistler wave identified in the data processed using peak suppression are very similar to those identified in the data processed using the VEX method. The most significant differences are during the interval between 01:43:18 and 01:43:26 where some whistlers were falsely identified in the reaction wheel frequency band $(32-40 \mathrm{~Hz})$ in the results from the VEX method but not in the peak suppression results. One of the reasons it is so important to accurately remove the reaction wheel noise is that it can mimic whistler waves. Power in the transverse and compressional modes can be used to identify false positives. The power in the transverse waves should be larger than that in the compressional waves for whistler waves. One example is between 01:43:24 and 01:43:25 where the compressional wave power is larger than the transverse wave power indicating that is was a false positive in the VEX method results. The result is that individual packets of whistler waves are identified in the peak suppression results whereas whistler waves were identified continuously from 01:43:19 to 01:43:34 in the VEX results.

Residual reaction wheel noise shows up in the dynamic spectra in figure 14 as a band between $32 \mathrm{~Hz}$ and $40 \mathrm{~Hz}$. The differences in the processing results are most distinctive in the ellipticity (figures $14(\mathrm{~B})$ and $(\mathrm{G})$ ) and propagation angle (figures $14(\mathrm{C})$ and $(\mathrm{H})$ ) results. The differences between the two methods are shown in figure 15 . The time series in panels a-d are the same as the red lines in figure 13. The largest differences are between 01:43:18 and 01:43:26. The absolute difference in the dynamic spectra from the two methods for the three components that are used to identify the whistler signatures are 

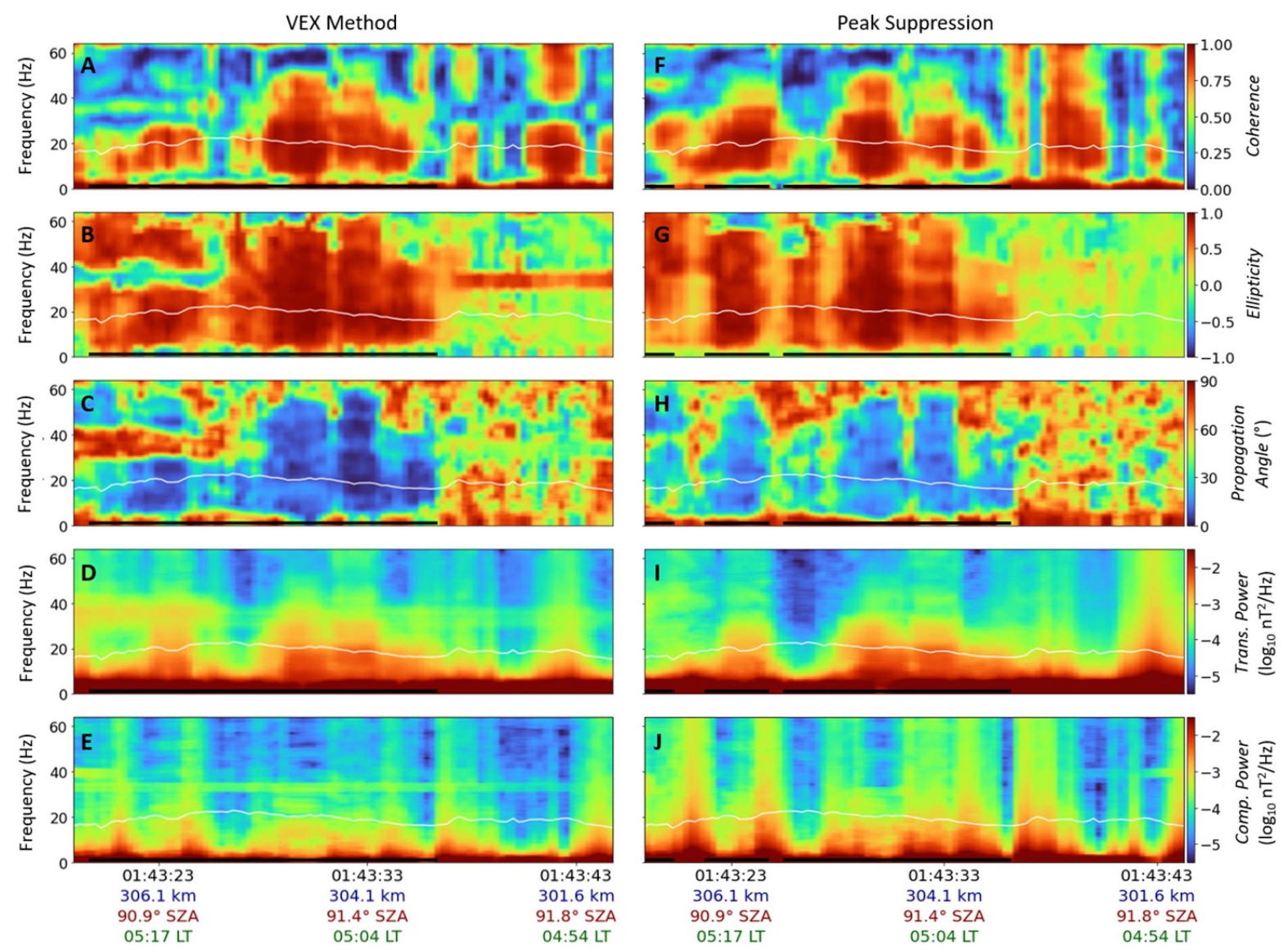

Figure 14. Dynamic spectra of the Venus Express magnetometer measurements for 9 June 2006, 01:43:18-01:43:45. (A)-(E) Results using data processed with the VEX method. (F)-(J) Results for the same interval using peak suppression. (A), (F) Coherence of the fluctuations on the $\mathrm{x}-\mathrm{z}$ plane. (B), (G) Ellipticity, where blue indicates left-hand polarization and red indicates right-hand polarization. (C), (H) Propagation angle with respect to the background magnetic field. (D), (I) Power in the transverse wave components. (E), (J) Power in the compressional wave components. The white line in each plot shows the field magnitude $|\mathrm{B}|(\mathrm{nT} / \mathrm{Hz})$. The thick black line at the bottom of each panel indicates the times where whistler waves were identified.

shown in figures $15(\mathrm{E})-(\mathrm{G})$. The differences in the time series prior to 01:43:26 correspond to differences in residual noise in the reaction wheel frequency band in the dynamic spectra. There are also some differences after 01:43:37. During the interval between 01:43:26 and 01:43:37 the results from the two methods are nearly identical.

Based on both the time series results and the whistler wave analysis, the gradiometry peak suppression method was able to effectively remove the reaction wheel noise from the the $128 \mathrm{~Hz}$ magnetometer data for 9 June 2006. We were able to reproduce the timing, amplitude, ellipticity, propagation angle and wave power for the fluctuations that have been identified as whistler waves generated by lightning on Venus. Peak suppression results also show two distinct packets of whistler waves in agreement with the fluctuations in the time series shown in figure 13.

\section{Discussion}

The primary benefit of the gradiometry peak suppression technique is that frequencies of signals generated by the FS can be specifically targeted for removal instead of filtering all fluctuations within a given frequency band or above/below a given threshold in the sensor data. Additionally, as demonstrated in both the results from Model 1, as well as the results from processing the VEX data, multiple FS generated AC signals can be removed simultaneously from a given interval. The interval shown in figure 7 includes four spectral peaks with frequencies between 0.1 and $8 \mathrm{~Hz}$ that were identified in the differenced spectrum and removed from the observed spectrum. This demonstrates that the peak suppression method works well even when we have signals from multiple components combined in the total observed signal. AC signals are removed to an uncertainty $<0.4 \mathrm{nT}$, less than twice the instrument noise included in the model. In addition, there are multiple spacecraft AC frequencies contained in the VEX observations. All frequencies were effectively removed, as shown in the final difference field (figure 13).

The limit on the algorithm's ability to accurately remove a FS field is imposed by the difference between the FS field frequencies and the ambient field frequencies. In general, the frequencies contained in the ambient and FS fields must differ by more than $1 / d t$ since this determines the resolution of the frequencies in the FFT. It is important to make sure that the intervals selected are sufficiently long in duration to meet 


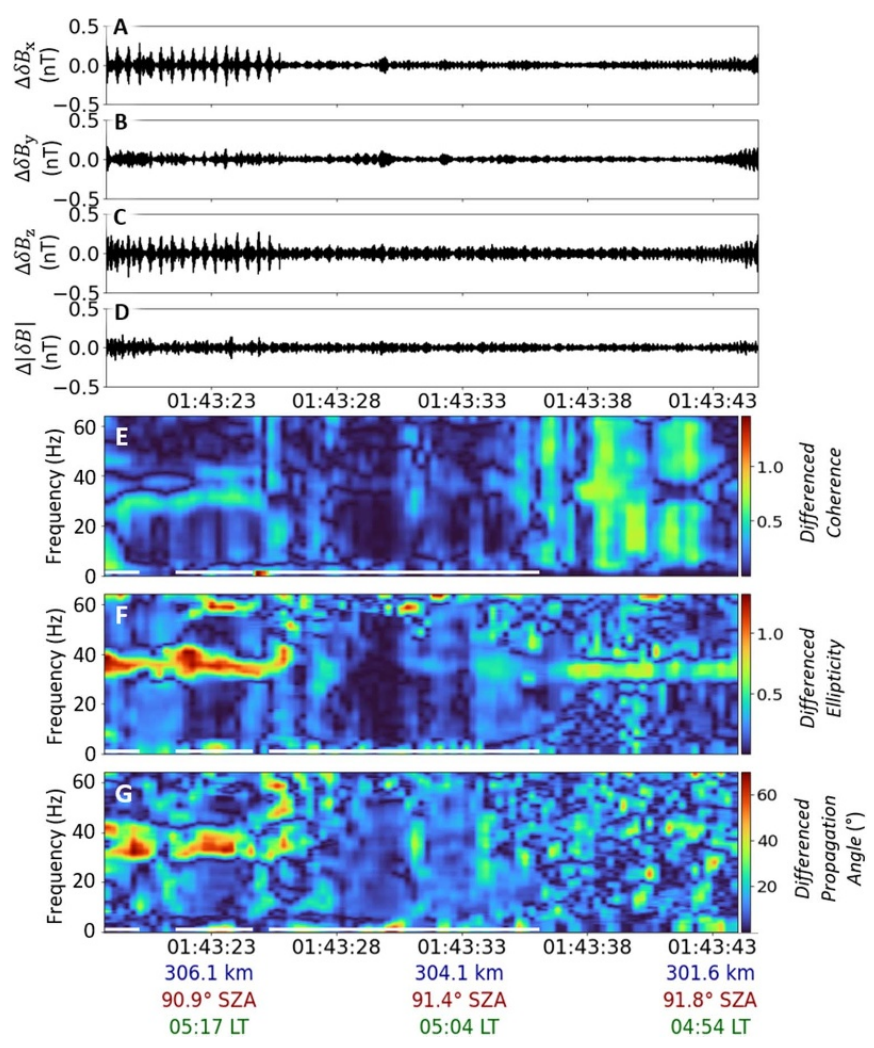

Figure 15. Differences in the time series and dynamic spectra results from VEX method processing and peak suppression processing for $(\mathrm{A}) \delta \mathrm{B}_{\mathrm{x}},(\mathrm{B}) \delta \mathrm{B}_{\mathrm{y}},(\mathrm{C}) \delta \mathrm{B}_{\mathrm{z}}$, (D) $|\delta \mathrm{B}|$, (E) coherence, (F) ellipticity, and $(\mathrm{G})$ propagation angle. Times where whistlers were identified are shown by the thick white line at the bottom of panels $(\mathrm{E})-(\mathrm{G})$.

the required frequency resolution for a given application. The duration of the intervals selected can be controlled by the specifications used for the upper and lower envelopes. The duration for the rolling maximum and minimum will determine the shortest interval that can be selected.

In Model 2, each burst of FS-generated fields has a duration of $60 \mathrm{~s}$, which results in a spectral resolution of $\sim 0.02 \mathrm{~Hz}$. For any spectral peaks in the FS field, there must not be an ambient field peak that falls with $\sim 0.04 \mathrm{~Hz}$ of that signal, or else both the ambient and spacecraft field contributions will be completely suppressed. Only FS signals with a frequency $1.75 \leqslant f \leqslant 1.95 \mathrm{~Hz}$, given a step size of $0.05 \mathrm{~Hz}$ in the frequencies tested, were identified and removed from the time series to within $0.6 \mathrm{nT}$. During the intervals with FS frequencies of $1.8 \mathrm{~Hz}$ and $1.9 \mathrm{~Hz}$, the error in field removal was $\sim 1.2 \mathrm{nT}$ while the error for the rest of the intervals was $<0.3 \mathrm{nT}$.

Another benefit of this method is that it is very flexible. The only requirements are that the gradiometer sensors be time synchronized and spacially separated enough for the FS AC signals to have different amplitudes at each sensor. Having the sensors further apart is ideal so that the differenced field is large. If the sensors are very close together then the differenced field will be at or near noise levels. There is no requirement for the FS fields to be dipolar at the sensors. The VEX spacecraft had sensors separated by $2 \mathrm{~m}$ with one very close to the spacecraft. This results in a very large differenced field. It also results in lot of stray fields being observed at the inboard sensor which were completely absent from the outboard sensor measurements. The peak suppression method performs as well in this case as it does with the modeled data shown in section 3 which were built with the assumption that the sensors are placed further from the spacecraft bus $(>1.4 \mathrm{~m})$.

Although the method was demonstrated here only for modeled data and for observations collected by VEX, application of the peak suppression method is not limited to spin stabilized spacecraft, nor is it limited to observations collected by fluxgate magnetometers. The only requirement is that the spacecraft carry a gradiometer consisting of two or more time synchronized instruments so that the differenced field can be used to determine the frequencies of the FS generated fields. Peak suppression could similarly be applied to measurements taken by search-coil or vector helium magnetometers if they are used in a gradiometer configuration. If the method is to be used on a spinning spacecraft the data should be de-spun prior to the application of the peak suppression method. This will eliminate the risk of distorting the spin related fluctuations if there are similar frequencies in the FS AC fields.

\section{Conclusions and future work}

Our frequency-domain gradiometry filtering can be used to remove FS magnetic fields from magnetic gradiometer data. Both the minimum frequency that can be removed and the minimum frequency resolution that can be achieved are determined by the frequency resolution of the FFT which is, in turn, determined by the duration of the interval being processed.

Using Model 1, we showed that the method is capable of removing concurrent signals from multiple FS sources at once. Figure 7 shows that the FS fluctuations are significantly reduced by the gradiometry peak suppression method with a resulting amplitude of $\sim 0.25 \mathrm{nT}$ in all components of the reconstructed ambient field (figures $7(\mathrm{~J})-(\mathrm{L})$ ). The resulting error in the calculations for the entire 30 min synthetic dataset compared to the original ambient field measurments used to build the model is $<0.6 \mathrm{nT}$.

Model 2 results indicate that the gradiometry peak suppression method is capable of removing FS generated peaks that are separated from the ambient field frequencies by greater than $0.05 \mathrm{~Hz}$. This is driven by the frequency resolution of the FFTs for the individual intervals where FS fields were identified which is, in turn, driven by the number of measurements in those intervals and the sampling frequency. Lower frequencies may be removed by using longer duration time series.

To validate the method on real data, we reprocessed $128 \mathrm{~Hz}$ magnetic field data from the VEX mission. We were able to show that the method is effective at identifying and removing the multiple spectral peaks generated by the reaction wheels on the spacecraft. The resulting spectra and wave forms are consistent with the fluctuations that have been identified as whistler waves generated by lightning in the Venusian atmosphere $[8,24,27]$.

Gradiometry peak suppression is a straightforward method that can autonomously identify and remove several FS 
generated signals in a single step. A list of discrete frequencies is constructed based on the frequencies that have an amplitude above the 98th percentile in the differenced field and each of those frequenices is removed from the observed spectra. There is no need to identify the true amplitude of the FS signals prior to removing them. If the fluctuations are strong enough to cause an observable peak in the field spectrum, they can be removed. The lower limit on the FS field amplitude that can be removed using this method has been identified as $\delta b=b_{n}$ using modeled data, where $b_{n}$ is the instrument noise. This is much lower than the limits on standard gradiometry methods which are typically several times the instrument noise.

Several possibilities exist for improving the technique. In cases where the ambient field frequency is too close to the spacecraft field frequency, it may be possible to use gradiometry peak suppression with coupling coefficients in frequency space [9] to determine the amplitude of the FS field at the inboard and outboard sensors. This could be used to determine how much of the signals to suppress. The trade off is that this would require accurately identifying the coupling coefficients which would increase the minimum amplitude required for signal identification. Continuing investigations into the best methods for integrating gradiometry peak suppression with coupling coefficients are underway. There are also cases where the FS fields have power over a wide and continuous range of frequencies. While peak suppression works best for well defined FS AC frequencies, it can select any number of frequencies for removal in a given window. However, the challenge in this situation is that, for a wide band of FS frequencies, ambient field frequencies are likely to be removed as well.

\section{Data availability statement}

All data that support the findings of this study are included within the article (and any supplementary files).

\section{Acknowledgments}

This work is supported by NASA Contract NNM16AA09, 'Psyche: Journey to a Metal World.'

Venus Express $128 \mathrm{~Hz}$ data is available through the Planetary Data Systems (PDS), DOI:10.17189/1522415. We would like to thank Tielong Zhang, VEX Magnetometer Principal Investigator, and the entire VEX magnetometer team for their efforts in collecting, processing and distributing the data.

Juno data were obtained through the PDS Juno Magnetometer Cruise archive, DOI:10.17189/1519716.

\section{ORCID iDs}

Jodie B Ream (D) https://orcid.org/0000-0002-9212-4291 Carol A Polanskey (D) https://orcid.org/0000-0001-67824408

Linda T Elkins-Tanton (D) https://orcid.org/0000-0003-40081098

\section{References}

[1] De Soria-santacruz M et al 2020 An approach to magnetic cleanliness for the psyche mission 2020 IEEE Aerospace Conf. pp 1-15

[2] Anderson B J, Zanetti L J, Lohr D H, Hayes J R, Acuna M H, Russell C T and Mulligan T 2001 In-flight calibration of the NEAR magnetometer IEEE Trans. Geosci. Remote Sens. 39 907-17

[3] Balogh A et al 2001 The cluster magnetic field investigation: overview of in-flight performance and initial results Ann. Geophys. 19 1207-17

[4] Carr C et al 2005 The double star magnetic field investigation: instrument design, performance and highlights of the first year's observations Ann. Geophys. 23 2713-32

[5] Carter D, Freesland D, Tadikonda S K, Kronenwetter J, Todirita M, Dahya M and Chu D 2016 Correcting GOES-R magnetometer data for stray fields ESA Workshop on Aerospace EMS, vol. 738 of ESA Special Publication p 59

[6] Georgescu E, Auster H U, Takada T, Gloag J, Eichelberger H, Fornaçon K H, Brown P, Carr C M and Zhang T L 2008 Modified gradiometer technique applied to double star (TC-1) Adv. Space Res. 41 1579-84

[7] Pope S A, Zhang T L, Balikhin M A, Delva M, Hvizdos L, Kudela K and Dimmock A P 2011 Exploring planetary magnetic environments using magnetically unclean spacecraft: a systems approach to VEX MAG data analysis Ann. Geophys. 29 639-47

[8] Russell C T, Zhang T L, Strangeway R J, Wei H Y, Delva M and Magnes W 2008 Electromagnetic waves observed by Venus Express at periapsis: detection and analysis techniques Adv. Space Res. 41 113-7

[9] Ness N F, Behannon K W, Lepping R P and Schatten K H 1971 Use of two magnetometers for magnetic field measurements on a spacecraft J. Geophys. Res. 76 3564-73

[10] Antoine J P, Coron A and Dereppe J M 2000 Water peak suppression: time-frequency vs time-scale approach $J$. Magn. Reson. 144 189-94

[11] Chen J-H and Singer S 2007 Chapter 4-high-resolution magic angle spinning NMR spectroscopy The Handbook of Metabonomics and Metabolomics ed J C Lindon, J K Nicholson and E Holmes (Amsterdam: Elsevier Science B.V.) pp 113-47

[12] Glanzer S, Schrank E and Zangger K 2013 A general method for diagonal peak suppression in homonuclear correlated nmr spectra by spatially and frequency selective pulses $J$. Magn. Reson. 232 1-6

[13] Starck J-L and Murtagh F 1994 Image restoration with noise suppression using the wavelet transform Astron. Astrophys. 288 342-8

[14] Sezn M I, Teklap A M and Schaetzing R 1989 Automatic anatomically selective image enhancement in digital chest radiography IEEE Trans. Med. Imaging 8 154-62

[15] Kaiser J and Schafer R 1980 On the use of the i0-sinh window for spectrum analysis IEEE Trans Acous. Speech Signal Process. 28 105-7

[16] Polanskey C A, Elkins-Tanton L T, Bell J F, Lawrence D J, Marchi S, Park R S, Russell C T and Weiss B P 2017 Psyche mission: scientific models and instrument selection AGU Fall Meeting Abstracts vol 2017 pp P33G-02

[17] Elkins-Tanton L T et al 2020 Observations, meteorites, and models: a preflight assessment of the composition and formation of (16) Psyche J. Geophys. Res. (Planets) 125 e06296

[18] Connerney J E P et al 2017 The Juno magnetic field investigation Space Sci. Rev. 213 39-138

[19] Connerney J E P 2016 Juno mag calibrated data ss v1.0, jno-ss-3-fgm-cal-v1.0 (Available at: https://doi.org/ $10.17189 / 1519716)$ 
[20] Svedhem H, Titov D, Taylor F and Witasse O 2009 Venus Express mission J. Geophys. Res. (Planets) 114 E00B33

[21] Zhang T L et al 2006 Magnetic field investigation of the Venus plasma environment: expected new results from Venus Express Planet. Space Sci. 54 1336-43

[22] Zhang T L et al 2008 Initial Venus Express magnetic field observations of the Venus bow shock location at solar minimum Planet. Space Sci. 56 785-9

[23] Russell C T 1991 Venus Lightning Space Sci. Rev. 55 317-56

[24] Russell C T, Leinweber H, Hart R A, Wei H Y, Strangeway R J and Zhang T L 2013 Venus Express observations of ULF and ELF waves in the Venus ionosphere: wave properties and sources Icarus 226 1527-37

[25] Means J D 1972 Use of the three-dimensional covariance matrix in analyzing the polarization properties of plane waves J. Geophys. Res. 77 5551-9

[26] Russell C T, Luhmann J G and Strangeway R J 2016 Time series analysis techniques Space Physics: An Introduction (Cambridge: Cambridge University) Ch. A.4, pp 450-4

[27] Hart R A, Russell C T R and Zhang T L 2021 Statistical study of lightning-generated whistler-mode waves observed by Venus Express 